\title{
Quasilinear parabolic equations with $p(x)$-Laplacian diffusion terms and nonlocal boundary conditions
}

\author{
Abita Rahmoune and Benyattou Benabderrahmane
}

\begin{abstract}
In this study, we prove the existence of local solution for a quasi linear generalized parabolic equation with nonlocal boundary conditions for an elliptic operator involving the variable-exponent nonlinearities, using Faedo-Galerkin arguments and compactness method.
\end{abstract}

Mathematics Subject Classification (2010): 35K20, 35K59, 35B45, 35D30.

Keywords: Nonlocal boundary conditions, quasi-linear parabolic equations, generalized Lebesgue space and Sobolev spaces with variable exponents.

\section{Introduction}

Let $\Omega$ be a bounded domain in $\mathbb{R}^{n}, n \geq 2$ with a smooth boundary $\Gamma=\partial \Omega$. We consider the following quasi linear parabolic equations with nonlocal boundary conditions:

$$
\begin{gathered}
\frac{\partial u}{\partial t}-\sum_{i=1}^{n} \frac{\partial}{\partial x_{i}}\left(|u|^{p(x)-2} \frac{\partial u}{\partial x_{i}}\right)+|u|^{p(x)-2} u=f(x, t) \text { in } Q_{T}=\Omega \times(0, T), \\
u(x, 0)=u_{0}(x), \quad x \in \Omega, \\
u(x, t)=\int_{\Omega} K(x, y) u(y, t) d y, \quad x \in \Gamma, t \in(0, T),
\end{gathered}
$$

where the exponent $p(\cdot)$ is a given measurable function on $\bar{\Omega}$ such that:

$$
2 \leq n<p_{1} \leq p(x) \leq p_{2} \leq \infty,
$$

where

$$
p_{2}=e s s \sup _{x \in \Omega}(x), \quad p_{1}=e s s \inf _{x \in \Omega} p(x) .
$$


We also assume that $p(\cdot)$ satisfies the following Zhikov-Fan uniform local continuity condition :

$$
|p(x)-p(y)| \leq \frac{M}{|\log | x-y||}, \text { for all } x, y \text { in } \Omega \text { with }|x-y|<\frac{1}{2}, M>0 .
$$

In recent years, many authors have paid attention to the study of nonlinear hyperbolic, parabolic and elliptic equations with nonstandard growth condition. For instance, modeling of physical phenomena such as flows of electro-rheological fluids or fluids with temperature-dependent viscosity, thermoelasticity, nonlinear viscoelasticity, filtration processes through a porous media and image processing. More details on these problems can be found in $[5,8,1,3,4,15,17,18]$ and references therein.

Constant exponent. In $(1.1)$, when $p(\cdot)=p$ is constant, local, global existence and long-time behavior have been considered by many authors.

For instance, in the absence of the term $|u|^{p-2} u$ and when the kernel datum function $K(x, y)=0$, using the compactness method and Faedo-Galerkin techniques, the existence and uniqueness of a weak solution has been proved see [16].

Baili Chen in [7] generalized the result of Lions to the situation when the presence of $|u|^{p-2} u$ and when $K(x, y) \neq 0$ in problem (1.1), applying exactly the same technique introduced in [16, Problème 12, page 140.], the author by constructing the approximate Galerkin solution, he proved the existence of generalized solution, the uniqueness questions are still open.

Problem (1.1)-(1.3) is the extension of the problems in Lion's book [16, p.140] in which the boundary conditions are homogeneous and in [7] in which the variableexponent is constant. The uniqueness questions in problem (1.1)-(1.3) are more complicated than in [7] and are still open.

The main difficulty of this problem, concerns the weak converging approximate solution, is related to the presence of the quasilinear terms in (1.1) in the variableexponent.

In this paper a class of quasi linear generalized parabolic equation with nonlocal boundary conditions for an elliptic operator involving the variable-exponent nonlinearities was considered. Hence by using Faedo-Galerkin arguments and compactness method as in [16], we will show the local existence of problem (1.1)-(1.3).

\section{Preliminaries}

In this section we list and recall some well-known results and facts from the theory of the Sobolev spaces with variable exponent. (For the details see $[9,11,10$, $12,13,14])$.

Throughout the rest of the paper we assume that $\Omega$ is a bounded domain of $\mathbb{R}^{n}$, $n \geq 2$ with smooth boundary $\Gamma$, Let $p: \bar{\Omega} \rightarrow[1, \infty]$ be a measurable function. We denote by $L^{p(\cdot)}(\Omega)$ the set of measurable functions $u$ on $\Omega$ such that

$$
A_{p(\cdot)}(u)=\int_{\Omega}|u(x)|^{p(x)} d x<\infty .
$$


The variable-exponent space $L^{p(\cdot)}(\Omega)$ equipped with the Luxemburg norm

$$
\|u\|_{p(\cdot), \Omega}=\|u\|_{p(\cdot)}=\|u\|_{L^{p(\cdot)}(\Omega)}=\inf \left\{\lambda>0, A_{p(\cdot)}\left(\frac{u}{\lambda}\right) \leq 1\right\}
$$

is a Banach space.

In general, variable-exponent Lebesgue spaces are similar to classical Lebesgue spaces in many aspects, see the first discussed the $L^{p(x)}(\Omega)$ spaces and $W^{k, p(x)}(\Omega)$ spaces by Kovàcik and Rákosnik in [14].

Let us list some properties of the spaces $L^{p(\cdot)}(\Omega)$ which will be used in the study of the problem (1.1)-(1.3).

- It follows directly from the definition of the norm that (see [9]),

$$
\min \left(\|u\|_{p(\cdot)}^{p_{1}},\|u\|_{p(\cdot)}^{p_{2}}\right) \leq A_{p(\cdot)}(u) \leq \max \left(\|u\|_{p(\cdot)}^{p_{1}},\|u\|_{p(\cdot)}^{p_{2}}\right) .
$$

- Let $p, q, s \geq 1$ be measurable functions defined on $\bar{\Omega}$ such that

$$
\frac{1}{s(x)}=\frac{1}{p(x)}+\frac{1}{q(x)}, \text { for a.e. } x \in \Omega \text {. }
$$

if $u \in L^{p(\cdot)}(\Omega), v \in L^{q(\cdot)}(\Omega)$ then $u . v \in L^{s(\cdot)}(\Omega)$ and the following generalized Hölder inequality

holds.

$$
\|u v\|_{s(\cdot)} \leq 2\|u\|_{p(\cdot)}\|v\|_{q(\cdot)} .
$$

Let us consider the following variable-exponent Lebesgue Sobolev space (see [9]), $W^{1, p(\cdot)}(\Omega)=\left\{v \in L^{p(\cdot)}(\Omega)\right.$ : such that $|\nabla v|$ exists and $\left.|\nabla v| \in L^{p(\cdot)}(\Omega)\right\}$.

This space is a Banach space with respect to the norm

$$
\|u\|_{W_{0}^{1, p(\cdot)}(\Omega)}=\|u\|_{p(\cdot)}+\sum_{i}\left\|\nabla u_{i}\right\|_{p(\cdot)} \cdot
$$

Furthermore, we set $W_{0}^{1, p(\cdot)}(\Omega)$, to be the closure of $C_{0}^{\infty}(\Omega)$ in $W^{1, p(\cdot)}(\Omega)$. Here we note that the space $W_{0}^{1, p(\cdot)}(\Omega)$ is usually defined in a different way for the variable exponent case. However (see Diening et al [9]), both definitions are equivalent under (1.5). The $\left(W_{0}^{1, p(\cdot)}(\Omega)\right)^{\prime}$ is the dual space of $W_{0}^{1, p(\cdot)}(\Omega)$ with respect to the inner product in $L^{2}(\Omega)$ and is defined as $W^{-1, p^{\prime}(\cdot)}(\Omega)$, in the same way as the classical Sobolev spaces, where $\frac{1}{p(.)}+\frac{1}{p \prime(\cdot)}=1$, the function $p^{\prime}(\cdot)$ is called the dual variable exponent of $p(\cdot)$.

- Let $p, q: \Omega \rightarrow[1,+\infty)$ be measurable functions satisfying condition (1.5). If $p(x) \leq q(x)$ almost everywhere in $\Omega$, then the embedding $L^{q(\cdot)}(\Omega) \hookrightarrow L^{p(\cdot)}(\Omega)$ is continuous.

Lemma 2.1. ([9]) Let $\Omega$ be a bounded domain in $\mathbb{R}^{n}, n \geq 1$ with a smooth boundary $\Gamma=\partial \Omega, p(\cdot)$ is a given measurable function on $\bar{\Omega}$ satisfy conditions (1.5) and $q=$ const $\geq 1$. If $q \leq p(x)$ a.e. in $\Omega$, then

$$
\|v\|_{q} \leq C_{q, \Omega}\|v\|_{p(\cdot)} \text { with the constant } C_{q, \Omega}=(1+|\Omega|)^{\frac{1}{q}} .
$$




\section{Notations and preliminaries}

In this article, on $f, u_{0}$ and $K(x, y)$ we make the following assumptions

$$
\begin{gathered}
f \in L^{p_{2}^{\prime}}\left(0, T ; L^{p_{2}^{\prime}}(\Omega)\right), \frac{1}{p_{2}}+\frac{1}{p_{2}^{\prime}}=1, \\
u_{0} \in L^{\infty}(\Omega), \\
\text { for any } x \in \Gamma, K(x)<\infty, K_{i}(x)<\infty, \\
\sum_{i=1}^{n} \int_{\Gamma} K(x)^{p_{2}-1} K_{i}(x) d \Gamma<\infty, \sum_{i=1}^{n} \int_{\Gamma} K(x)^{p_{1}-1} K_{i}(x) d \Gamma<\infty, \\
\gamma=\max \left(\begin{array}{c}
C_{p_{1}, \Omega}^{p_{2}}\left(\sum_{i=1}^{n} \int_{\Gamma} K(x)^{p_{2}-1} K_{i}(x) d \Gamma\right), \\
\left(C_{p_{1}, \Omega}^{p_{1}} \sum_{i=1}^{n} \int_{\Gamma} K(x)^{p_{1}-1} K_{i}(x) d \Gamma\right)
\end{array}\right) \leq \frac{p_{1}-1}{p_{2}},
\end{gathered}
$$

for any $x \in \Gamma$, where

$$
K(x)=\left(\int_{\Omega}|K(x, y)|^{p_{1}^{\prime}} d y\right)^{\frac{1}{p_{1}^{\prime}}}:
$$

norm of $k(x, y)$ in $L^{p_{1}^{\prime}}(\Omega)$ with respect to $y, \frac{1}{p_{1}}+\frac{1}{p_{1}^{\prime}}=1$

$$
K_{i}(x)=\left(\sum_{i=1}^{n} \int_{\Omega}\left|\frac{\partial}{\partial x_{i}} K(x, y)\right|^{p_{1}^{\prime}} d y\right)^{\frac{1}{p_{1}^{\prime}}}:
$$

norm of $\frac{\partial}{\partial x_{i}} k(x, y)$ in $L^{p_{1}^{\prime}}(\Omega)$ with respect to $y, \frac{1}{p_{1}}+\frac{1}{p_{1}^{\prime}}=1$

and $C_{p_{1}, \Omega}$ defined in $(2.1)$.

Moreover, we assume that

$$
r>\frac{n}{2}+2
$$

Let

$$
\alpha_{1}=\left(\frac{p_{2}}{p_{1}}\left(\gamma+\frac{1}{p_{2}}\right)\right)^{\frac{p_{2}}{p_{1}-p_{2}}} .
$$

We define the polynomial $Q$ by

$$
Q(\alpha)=\min \left(\alpha, \alpha^{\frac{p_{1}}{p_{2}}}\right)-\left(\gamma+\frac{1}{p_{2}}\right) \max \left(\alpha, \alpha^{\frac{p_{1}}{p_{2}}}\right) \quad \forall \alpha \in[0,+\infty] .
$$

Let

$$
h(\alpha)=\alpha^{\frac{p_{1}}{p_{2}}}-\left(\gamma+\frac{1}{p_{2}}\right) \alpha .
$$

Notice that $h(\alpha)=Q(\alpha)$, for $1 \leq \alpha \leq \infty$. It is easy to check that the function $h(\alpha)$ is increasing for $1 \leq \alpha<\alpha_{1}$ and decreasing for $\alpha_{1}<\alpha \leq+\infty$, where $\alpha_{1}$ is its unique local maximum defined by (3.7). We will assume that:

$$
1 \leq\left\|u_{0}\right\|_{p(\cdot)}^{p_{2}}=\alpha_{0}<\alpha_{1}
$$


and

$$
\frac{1}{2}\left|u_{0}\right|^{2}+C_{2, \Omega}^{\frac{p_{2}}{p_{2}-1}} \frac{p_{2}-1}{p_{2}}|\Omega|^{\frac{1}{2}} \int_{0}^{T}|f|_{p_{2}}^{\frac{p_{2}}{p_{2}-1}} d t<\int_{0}^{T} Q\left(\alpha_{1}\right) d t .
$$

The classical formulation of the problem is as follows. Find a displacement field $u$ : $\Omega \times(0, T) \rightarrow \mathbb{R}$, such that:

$$
\begin{gathered}
\left(u^{\prime}, v\right)-\left(\sum_{i=1}^{n} \frac{\partial}{\partial x_{i}}\left(|u|^{p(x)-2} \frac{\partial u}{\partial x_{i}}\right), v\right)+\left(|u|^{p(x)-2} u, v\right)=(f, v), \forall v \in V \\
u(x, 0)=u_{0}(x), \quad x \in \Omega
\end{gathered}
$$

Where

$$
V=\left\{v \in H^{r}(\Omega): v(x)=\int_{\Omega} K(x, y) v(y) d y \text { for } x \in \Gamma\right\},
$$

With assumption (1.4)-(3.6), using Sobelev embedding theorems, see [2], we have

$$
H^{r}(\Omega) \hookrightarrow W^{2, p_{2}}(\Omega) \hookrightarrow W^{1, p_{2}}(\Omega) \hookrightarrow L^{p_{2}}(\Omega) \hookrightarrow L^{2}(\Omega)
$$

It is easy to see that $V$ is a subspace of $H^{r}(\Omega)$.

Whenever it doesn't cause a confusion, we use the following shorthand notations:

$L^{q}(\Omega): L^{q}$ space defined on $\Omega ;|\cdot|_{q}=|\cdot|_{q, \Omega}$ : norm in $L^{q}(\Omega) ;|\cdot|_{q, \Gamma}$ : norm in $L^{q}(\Gamma)$; $H^{-r}(\Omega)$ : dual space of $H^{r}(\Omega) ;|\cdot|_{H^{-r}(\Omega)}$ norm in $H^{-r}(\Omega) ; C$ : nonnegative constant which may take different values on each occurrence.

\section{Local existence}

Theorem 4.1. Under hypothesis (1.4)-(3.9), for any finite $T>0$, the problem (1.1)(1.3) admits a weak solution $u$ such that

$$
\begin{gathered}
u \in L^{\infty}\left(0, T ; L^{2}(\Omega)\right) \cap C\left([0, T] ; H^{-r}(\Omega)\right) \cap L^{p(\cdot)}(\Omega \times(0, T)), \\
\frac{\partial u}{\partial t} \in L^{p_{2}^{\prime}}\left(0, T ; H^{-r}(\Omega)\right), \\
|u|^{\frac{p(\cdot)-2}{2}} u \in L^{2}\left(0, T ; H^{1}(\Omega)\right),
\end{gathered}
$$

for all $v \in V$ and a.e. $t \in[0, T]$,

$$
\begin{gathered}
\left(u^{\prime}, v\right)-\left(\sum_{i=1}^{n} \frac{\partial}{\partial x_{i}}\left(|u|^{p(x)-2} \frac{\partial u}{\partial x_{i}}\right), v\right)+\left(|u|^{p(x)-2} u, v\right)=(f, v), \\
u(x, 0)=u_{0}(x), x \in \Omega .
\end{gathered}
$$

Proof. Since $V$ is a subspace of $H^{r}(\Omega)$ which is separable. We can choose a countable set of distinct basis elements $w_{j}(j=1,2, \ldots)$ which generate $V$ and are orthonormal in $L^{2}(\Omega)$. Let $V_{m}$ be the subspace of $V$ generated by the first $m$ elements: $w_{1}, w_{2}, \ldots, w_{m}$. We search $u$ of the form:

$$
u_{m}(x, t)=\sum_{i=1}^{m} K_{i m}(t) w_{i}(x)
$$


satisfying:

with

$$
\left\{\begin{array}{c}
\left(u_{m}^{\prime}, w_{j}\right)-\left(\sum_{i=1}^{n} \frac{\partial}{\partial x_{i}}\left(\left|u_{m}\right|^{p(x)-2} \frac{\partial u_{m}}{\partial x_{i}}\right), w_{j}\right) \\
+\left(\left|u_{m}\right|^{p(x)-2} u_{m}, w_{j}\right)=\left(f(t), w_{j}\right), \quad 1 \leq j \leq m, \\
u_{m}(0)=u_{0 m} .
\end{array}\right.
$$

$$
u_{0 m}=\sum_{i=1}^{m} \alpha_{i m} w_{i} \longrightarrow u_{0} \text { when } m \longrightarrow \infty \text { in } L^{p(\cdot)}(\Omega) .
$$

Integrating by parts on the second term of left-hand side of (4.6), we have

$$
\left\{\begin{array}{c}
\left(u_{m}^{\prime}, w_{j}\right)+\left(\sum_{i=1}^{n}\left(\left|u_{m}\right|^{p(x)-2} \frac{\partial u_{m}}{\partial x_{i}}\right), \frac{\partial}{\partial x_{i}} w_{j}\right)+\left(\left|u_{m}\right|^{p(x)-2} u_{m}, w_{j}\right) \\
=\int_{\Gamma}\left|u_{m}\right|^{p(x)-2} \frac{\partial u_{m}}{\partial x_{i}} w_{j} d \Gamma+\left(f(t), w_{j}\right), \quad 1 \leq j \leq m, \\
u_{m}(0)=u_{0 m} .
\end{array}\right.
$$

By Peano's Theorem, for every finite $m$ the problem (4.6), (4.8) has a solution on $\left(0, T_{m}\right)$ for each $m$. The following estimates permit us to confirm that $T_{m}$ is independent of $m$.

a) A priori estimates

Multiplying the equation (4.8) by $K_{j m}(t)$, summing over $j=1, \ldots, m$, we obtain

$$
\begin{gathered}
\frac{1}{2} \frac{d}{d t}\left|u_{m}(t)\right|^{2}+\sum_{i=1}^{n} \int_{\Omega} \frac{4}{p^{2}(x)}\left(\frac{\partial}{\partial x_{i}}\left(\left|u_{m}\right|^{\frac{p(x)-2}{2}} u_{m}\right)\right)^{2} d x+\int_{\Omega}\left|u_{m}\right|^{p(x)} d x \\
=\int_{\Gamma}\left|u_{m}\right|^{p(x)-2} \frac{\partial u_{m}}{\partial x_{i}} u_{m}(t) d \Gamma+\left(f(t), u_{m}\right)
\end{gathered}
$$

Integrating on $(0, T)$ on both sides of $(4.9)$, we get

$$
\begin{aligned}
\frac{1}{2}\left|u_{m}(T)\right|^{2} & +\int_{0}^{T} \sum_{i=1}^{n} \int_{\Omega} \frac{4}{p^{2}(x)}\left(\frac{\partial}{\partial x_{i}}\left(\left|u_{m}\right|^{\frac{p(x)-2}{2}} u_{m}\right)\right)^{2} d x d t \\
& +\int_{0}^{T} \min \left(\left.|| u_{m}\right|_{p(\cdot)} ^{p_{2}},|| u_{m}||_{p(\cdot)}^{p_{1}}\right) d t \\
\leq\left.\int_{0}^{T} \int_{\Gamma}|| u_{m}\right|^{p(x)-2} & \left.\frac{\partial u_{m}}{\partial x_{i}} u_{m}(t)\left|d \Gamma d t+\int_{0}^{T}\right|\left(f(t), u_{m}\right)\left|d t+\frac{1}{2}\right| u_{0 m}\right|^{2} .
\end{aligned}
$$

The second term in the right-hand side of (4.10) can be estimated as follows

$$
\begin{gathered}
\left|\left(f(t), u_{m}\right)\right| \leq|f|_{2}\left|u_{m}\right|_{2} \leq C_{2, \Omega}|f|_{2}\left\|u_{m}\right\|_{p(\cdot)} \quad \text { (holder's inequality) and (2.1) } \\
\leq C_{2, \Omega}^{\frac{p_{2}}{p_{2}-1}} \frac{p_{2}-1}{p_{2}}|f|_{2}^{\frac{p_{2}}{p_{2}-1}}+\frac{1}{p_{2}}\left\|u_{m}\right\|_{p(\cdot)}^{p_{2}} \quad \text { (Young's inequality) } \\
\leq C_{2, \Omega}^{\frac{p_{2}}{p_{2}-1}} \frac{p_{2}-1}{p_{2}}|\Omega|^{\frac{1}{2}}|f|_{p_{2}}^{\frac{p_{2}}{p_{2}-1}}+\frac{1}{p_{2}} \max \left(\left\|u_{m}\right\|_{p(\cdot)}^{p_{2}},\left\|u_{m}\right\|_{p(\cdot)}^{p_{1}}\right)
\end{gathered}
$$

Next, we estimate first term in the right-hand side of (4.10) using (2.1): For $x \in \Gamma$, we have

$$
\left|u_{m}(x, t)\right| \leq K(x)\left|u_{m}\right|_{p_{1}} \leq C_{p_{1}, \Omega} K(x)\left\|u_{m}\right\|_{p(\cdot)} .
$$


Similarly, for $x \in \Gamma$ we have

$$
\left|\sum_{i=1}^{n} \frac{\partial}{\partial x_{i}} u_{m}(x, t)\right| \leq K_{i}(x)\left|u_{m}\right|_{p_{1}} \leq C_{p_{1}, \Omega} K_{i}(x)\left\|u_{m}\right\|_{p(\cdot)}
$$

Then using holder's inequality and assumptions (3.3) and (3.5), we have:

$$
\begin{gathered}
\left.\left.\sum_{i=1}^{n} \int_{\Gamma}|| u_{m}\right|^{p(x)-2} \frac{\partial u_{m}}{\partial x_{i}} u_{m}(t)\left|d \Gamma \leq \sum_{i=1}^{n} \int_{\Gamma}\right| u_{m}\right|^{p(x)-1}\left|\frac{\partial u_{m}}{\partial x_{i}}\right| d \Gamma \\
\leq \max \left(\sum_{i=1}^{n} \int_{\Gamma}\left|u_{m}\right|^{p_{2}-1}\left|\frac{\partial u_{m}}{\partial x_{i}}\right| d \Gamma, \sum_{i=1}^{n} \int_{\Gamma}\left|u_{m}\right|^{p_{1}-1}\left|\frac{\partial u_{m}}{\partial x_{i}}\right| d \Gamma\right) \\
\leq \max \left(\begin{array}{c}
C_{p_{1}, \Omega}^{p_{2}} \sum_{i=1}^{n} \int_{\Gamma} K(x)^{p_{2}-1}\left\|u_{m}\right\|_{p(\cdot)}^{p_{2}-1} K_{i}(x)\left\|u_{m}\right\|_{p(\cdot)} d \Gamma, \\
C_{p_{1}, \Omega}^{p_{1}} \sum_{i=1}^{n} \int_{\Gamma} K(x)^{p_{1}-1}\left\|u_{m}\right\|_{p(\cdot)}^{p_{1}-1} K_{i}(x)\left\|u_{m}\right\|_{p(\cdot)} d \Gamma
\end{array}\right) \\
=\max \left(\begin{array}{c}
C_{p_{1}, \Omega}^{p_{2}}\left(\sum_{i=1}^{n} \int_{\Gamma} K(x)^{p_{2}-1} K_{i}(x) d \Gamma\right)\left\|u_{m}\right\|_{p(\cdot)}^{p_{2}}, \\
C_{p_{1}, \Omega}^{p_{1}, \Omega}\left(\sum_{i=1}^{n} \int_{\Gamma} K(x)^{p_{1}-1} K_{i}(x) d \Gamma\right)\left\|u_{m}\right\|_{p(\cdot)}^{p_{1}}
\end{array}\right) \\
\leq \max \left(\begin{array}{c}
C_{p_{1}, \Omega}^{p_{2}}\left(\sum_{i=1}^{n} \int_{\Gamma} K(x)^{p_{2}-1} K_{i}(x) d \Gamma\right), \\
\left(C_{p_{1}, \Omega}^{p_{1}} \sum_{i=1}^{n} \int_{\Gamma} K(x)^{p_{1}-1} K_{i}(x) d \Gamma\right)
\end{array}\right) \\
\times \max \left(\left\|u_{m}\right\|_{p(\cdot)}^{p_{2}},\left\|u_{m}\right\|_{p(\cdot)}^{p_{1}}\right)
\end{gathered}
$$

This implies that

$$
\begin{gathered}
\frac{1}{2}\left|u_{m}(t)\right|^{2}+\int_{0}^{T} \sum_{i=1}^{n} \int_{\Omega} \frac{4}{p^{2}(x)}\left(\frac{\partial}{\partial x_{i}}\left(\left|u_{m}\right|^{\frac{p(x)-2}{2}} u_{m}\right)\right)^{2} d x d t+\int_{0}^{T} Q\left(\left.|| u_{m}\right|_{p(\cdot)} ^{p_{2}}\right) d t \\
\leq \frac{1}{2}\left|u_{0 m}\right|^{2}+C_{2, \Omega}^{\frac{p_{2}}{p_{2}-1}} \frac{p_{2}-1}{p_{2}}|\Omega|^{\frac{1}{2}} \int_{0}^{T}|f|_{p_{2}}^{\frac{p_{2}}{p_{2}-1}} d t
\end{gathered}
$$

at this step we will assume that $Q\left(\left\|u_{m}\right\|_{p(\cdot)}^{p_{2}}\right) \geq 0$, so from (3.9) and (4.13), we have the following a priori estimates:

$$
\begin{gathered}
\left|u_{m}\right| \leq C(C \text { is independent of } m) ; \\
\int_{0}^{T} \sum_{i=1}^{n} \int_{\Omega} \frac{4}{p^{2}(x)}\left(\frac{\partial}{\partial x_{i}}\left(\left|u_{m}\right|^{\frac{p(x)-2}{2}} u_{m}\right)\right)^{2} d x d t \leq C(C \text { independent of } m) .
\end{gathered}
$$

So the solution $u_{m}(t)$ of problem (1.1)-(1.3) exists on $[0, T]$ for each $m$, and

$$
\begin{gathered}
u_{m} \text { is bounded in } L^{\infty}\left(0, T ; L^{2}(\Omega)\right) ; \\
\left|u_{m}\right|^{\frac{p(\cdot)-2}{2}} u_{m} \text { is bounded in } L^{2}\left(0, T ; H^{1}(\Omega)\right)
\end{gathered}
$$

Claim 4.2. There exists an integer $N$ such that

$$
\left\|u_{m}\right\|_{p(\cdot)}^{p_{2}}<\alpha_{1} \quad \forall t \in\left[0, T_{m}\right) \quad m>N .
$$


Proof of the Claim. Suppose (4.17) false. Then for each $m>N$, there exists $t \in$ $\left[0, T_{m}\right)$ such that $\left\|u_{m}(t)\right\|_{p(\cdot)}^{p_{2}} \geq \alpha_{1}$. We note that from (3.8) and (4.7) there exists $N_{0}$ such that

$$
1 \leq\left\|u_{m}(0)\right\|_{p(\cdot)}^{p_{2}}<\alpha_{1} \quad \forall m>N_{0}
$$

Then by continuity there exists a first $T_{m}^{*} \in\left(0, T_{m}\right)$ such that

$$
\left\|u_{m}\left(T_{m}^{*}\right)\right\|_{p(\cdot)}^{p_{2}}=\alpha_{1}
$$

from where

$$
Q\left(\left\|u_{m}\right\|_{p(\cdot)}^{p_{2}}\right)=h\left(\left\|u_{m}(t)\right\|_{p(\cdot)}^{p_{2}}\right) \geq 0 \quad \forall t \in\left[0, T_{m}^{*}\right] .
$$

Now from (3.9) and (4.13), there exist $N>N_{0}$ and $\beta \in\left(1 ; \alpha_{1}\right)$ such that

$$
\begin{aligned}
0 & \leq \frac{1}{2}\left|u_{m}(t)\right|^{2}+\int_{0}^{t} \sum_{i=1}^{n} \int_{\Omega} \frac{4}{p^{2}(x)}\left(\frac{\partial}{\partial x_{i}}\left(\left|u_{m}\right|^{\frac{p(x)-2}{2}} u_{m}\right)\right)^{2} d x d s \\
& +\int_{0}^{t} Q\left(\left\|u_{m}\right\|_{p(\cdot)}^{p_{2}}\right) d s \leq \int_{0}^{t} Q(\beta) d s \quad \forall t \in\left[0, T_{m}^{*}\right], \quad \forall m>N
\end{aligned}
$$

Then the monotonicity of $Q$ implies that

$$
\left\|u_{m}(t)\right\|_{p(\cdot)}^{p_{2}} \leq \beta<\alpha_{1} \quad \forall t \in\left[0, T_{m}^{*}\right]
$$

and in particular, $\left\|u_{m}\left(T_{m}^{*}\right)\right\|_{p(\cdot)}^{p_{2}}<\alpha_{1}$, which is a contradiction to (4.18). And then the supposition $Q\left(\left\|u_{m}\right\|_{p(\cdot)}^{p_{2}}\right) \geq 0$ is true.

From (4.17) the solution $u_{m}(t)$ of problem (1.1)-(1.3) satisfies other of (4.16),

$$
u_{m} \text { is bounded in } L^{p(\cdot)}(\Omega \times(0, T)) .
$$

Lemma 4.3. Let $u_{m}$, constructed in (4.5), be the approximate solution of (1.1)-(1.3). Then

$$
\frac{\partial}{\partial t} u_{m}(t) \text { is bounded in } L^{p_{2}^{\prime}}\left(0, T ; H^{-r}(\Omega)\right)
$$

Proof. Let $v \in H^{r}(\Omega)$, from (4.6) we have

$$
\begin{gathered}
\left(\frac{\partial u_{m}(t)}{\partial t}, v\right)+\left(\sum_{i=1}^{n}\left(\left|u_{m}\right|^{p(x)-2} \frac{\partial u_{m}}{\partial x_{i}}\right), \frac{\partial}{\partial x_{i}} v\right)+\left(\left|u_{m}\right|^{p(x)-2} u_{m}, v\right) \\
=\sum_{i=1}^{n} \int_{\Gamma}\left|u_{m}\right|^{p(x)-2} \frac{\partial u_{m}}{\partial x_{i}} v d \Gamma+(f(t), v),
\end{gathered}
$$

The last term in the left-hand side can be estimated as follows:

$$
\begin{gathered}
\left|\left(\left|u_{m}\right|^{p(x)-2} u_{m}, v\right)\right| \leq\left.\left.|| u_{m}\right|^{p(x)-1}\right|_{p_{2}^{\prime}}|v|_{p_{2}} \leq\left.\left. C|| u_{m}\right|^{p(x)-1}\right|_{p^{\prime}(\cdot)}|v|_{p_{2}} \quad\left(p_{2}^{\prime} \leq p^{\prime}(\cdot) \leq p_{1}^{\prime}\right) \\
\leq C \max \left(\left(\int_{\Omega}\left|u_{m}\right|^{p(x)} d x\right)^{\frac{1}{p_{1}^{\prime}}},\left(\int_{\Omega}\left|u_{m}\right|^{p(x)} d x\right)^{\frac{1}{p_{2}^{\prime}}}\right)|v|_{p_{2}} \\
\leq C \max \left(\left(\int_{\Omega}\left|u_{m}\right|^{p(x)} d x\right)^{\frac{1}{p_{1}^{\prime}}},\left(\int_{\Omega}\left|u_{m}\right|^{p(x)} d x\right)^{\frac{1}{p_{2}^{\prime}}}\right)|v|_{H^{r}}
\end{gathered}
$$


Hence,

$$
\left.\left.|| u_{m}\right|^{p(\cdot)-2} u_{m}\right|_{H^{-r}(\Omega)} \leq C \max \left(\left(\int_{\Omega}\left|u_{m}\right|^{p(x)} d x\right)^{\frac{1}{p_{1}^{\prime}}},\left(\int_{\Omega}\left|u_{m}\right|^{p(x)} d x\right)^{\frac{1}{p_{2}^{\prime}}}\right)<\infty .
$$

The norm of $\left|u_{m}\right|^{p(\cdot)-2} u_{m}$ in $L^{p_{2}^{\prime}}\left(0, T ; H^{-r}(\Omega)\right)$ is bounded by

$$
C\left(\int_{0}^{T} \max \left(\left(\int_{\Omega}\left|u_{m}\right|^{p(x)} d x\right)^{\frac{p_{2}^{\prime}}{p_{1}^{\prime}}}, \int_{\Omega}\left|u_{m}\right|^{p(x)} d x\right)\right)^{\frac{1}{p_{2}^{\prime}}}<\infty
$$

Therefore, $\left|u_{m}\right|^{p(\cdot)-2} u_{m}$ is bounded in $L^{p_{2}^{\prime}}\left(0, T ; H^{-r}(\Omega)\right)$.

Next, we consider the term $\sum_{i=1}^{n} \int_{\Gamma}\left|u_{m}\right|^{p(x)-2} \frac{\partial u_{m}}{\partial x_{i}} v d \Gamma$ in the left-hand side of (4.21):

$$
\begin{gathered}
\left.\left.\left|\sum_{i=1}^{n} \int_{\Gamma}\right| u_{m}\right|^{p(x)-2} \frac{\partial u_{m}}{\partial x_{i}} v d \Gamma\left|\leq\left(\left.\left.\sum_{i=1}^{n}|| u_{m}\right|^{p(x)-2} \frac{\partial u_{m}}{\partial x_{i}}\right|_{p_{1}^{\prime}, \Gamma}\right)\right| v(t)\right|_{p_{1}, \Gamma} \\
=\left.\left.\sum_{i=1}^{n}|| \int_{\Omega} K(x, y) u_{m}(y) d y\right|^{p(x)-2} \int_{\Omega} \frac{\partial}{\partial x_{i}} K_{i}(x, y) u_{m}(y) d y\right|_{p_{1}^{\prime}, \Gamma} \\
\times\left|\int_{\Omega} K(x, y) v(y) d y\right|_{p_{1}, \Gamma} \\
\leq\left.\left.\left.\left. C \sum_{i=1}^{n}\left|K(x)^{p(x)-2} K_{i}(x)\right| u_{m}(y)\right|_{p_{1}} ^{p(x)-1}\right|_{p_{1}^{\prime}, \Gamma}|K(x)| v(y)\right|_{p_{1}}\right|_{p_{1}, \Gamma} \\
\leq C \sum_{i=1}^{n}\left|K(x)^{p(x)-2} K_{i}(x)\right|_{p_{1}^{\prime}, \Gamma}|K(x)|_{p_{1}, \Gamma}\left|u_{m}(y)\right|_{p_{1}}^{p(x)-1}|v(y)|_{p_{1}} \\
\leq C \max \left(\sum_{i=1}^{n}\left|K(x)^{p_{1}-2} K_{i}(x)\right|_{p_{1}^{\prime}, \Gamma}, \sum_{i=1}^{n}\left|K(x)^{p_{2}-2} K_{i}(x)\right|_{p_{1}^{\prime}, \Gamma}\right)|K(x)|_{p_{1}, \Gamma} \\
\times \max \left(\left|u_{m}(y)\right|_{p_{1}}^{p_{1}-1},\left|u_{m}(y)\right|_{p_{1}}^{p_{2}-1}\right)|v(y)|_{H^{r}(\Omega)} \\
\leq C \max \left(\sum_{i=1}^{n}\left|K(x)^{p_{1}-2} K_{i}(x)\right|_{p_{1}^{\prime}, \Gamma}, \sum_{i=1}^{n}\left|K(x)^{p_{2}-2} K_{i}(x)\right|_{p_{1}^{\prime}, \Gamma}\right)|K(x)|_{p_{1}, \Gamma} \\
\times \max \left(\left|u_{m}(y)\right|_{p(\cdot)}^{p_{1}-1},\left|u_{m}(y)\right|_{p_{(\cdot)}}^{p_{2}-1}\right)|v(y)|_{H^{r}}
\end{gathered}
$$

Therefore,

$$
\begin{gathered}
\left.\left.\left|\sum_{i=1}^{n} \int_{\Gamma}\right| u_{m}\right|^{p(x)-2} \frac{\partial u_{m}}{\partial x_{i}} d \Gamma\right|_{H^{-r}(\Omega)} \\
\leq C \max \left(\sum_{i=1}^{n}\left|K(x)^{p_{1}-2} K_{i}(x)\right|_{p_{1}^{\prime}, \Gamma}, \sum_{i=1}^{n}\left|K(x)^{p_{2}-2} K_{i}(x)\right|_{p_{1}^{\prime}, \Gamma}\right) \\
\times \max \left(\left|u_{m}(y)\right|_{p(\cdot)}^{p_{1}-1},\left|u_{m}(y)\right|_{p_{(\cdot)}^{p_{2}-1}}\right)|K(x)|_{p_{1}, \Gamma}<\infty .
\end{gathered}
$$


Then the norm of $\sum_{i=1}^{n} \int_{\Gamma}\left|u_{m}\right|^{p(x)-2} \frac{\partial u_{m}}{\partial x_{i}} d \Gamma$ in $L^{p_{2}^{\prime}}\left(0, T ; H^{-r}(\Omega)\right)$ is bounded by $C\left(\begin{array}{c}\int_{0}^{T} \max \left(\sum_{i=1}^{n}\left|K(x)^{p_{1}-2} K_{i}(x)\right|_{p_{1}^{\prime}, \Gamma}^{p_{2}^{\prime}}, \sum_{i=1}^{n}\left|K(x)^{p_{2}-2} K_{i}(x)\right|_{p_{1}^{\prime}, \Gamma}^{p_{2}^{\prime}}\right) \\ \times \max \left(\left|u_{m}(y)\right|_{p(\cdot)}^{\left(p_{1}-1\right) p_{2}^{\prime}},\left|u_{m}(y)\right|_{p_{(\cdot)}}^{\left(p_{2}-1\right) p_{2}^{\prime}}\right)|K(x)|_{p_{1}, \Gamma}^{p_{2}^{\prime}} d t\end{array}\right)^{\frac{1}{p_{2}^{\prime}}}<\infty$ Hence $\sum_{i=1}^{n} \int_{\Gamma}\left|u_{m}\right|^{p(x)-2} \frac{\partial u_{m}}{\partial x_{i}} d \Gamma$ is bounded in $L^{p_{2}^{\prime}}\left(0, T ; H^{-r}(\Omega)\right)$.

Next, we consider the second term in the left-hand side of (4.21). Integrating by parts gives

$$
\begin{gathered}
\left(\sum_{i=1}^{n}\left(\left|u_{m}\right|^{p(x)-2} \frac{\partial u_{m}}{\partial x_{i}}\right), \frac{\partial v}{\partial x_{i}}\right)=\int_{\Omega} \sum_{i=1}^{n} \frac{1}{p(x)-1}\left(\frac{\partial}{\partial x_{i}}\left(\left|u_{m}\right|^{p(x)-2} u_{m}\right)\right) \frac{\partial v}{\partial x_{i}} d x \\
=\int_{\Gamma} \sum_{i=1}^{n} \frac{1}{p(x)-1}\left|u_{m}\right|^{p(x)-2} u_{m} \frac{\partial v}{\partial x_{i}} d \Gamma-\int_{\Omega} \frac{1}{p(x)-1}\left|u_{m}\right|^{p(x)-2} u_{m} \Delta v d x
\end{gathered}
$$

First, we have

$$
\begin{gathered}
\left.\left.\left|\int_{\Gamma} \sum_{i=1}^{n} \frac{1}{p(x)-1}\right| u_{m}\right|^{p(x)-2} u_{m} \frac{\partial v}{\partial x_{i}} d \Gamma\left|\leq \frac{1}{p_{2}-1} \sum_{i=1}^{n}\right|\left|u_{m}\right|^{p(x)-2} u_{m}\right|_{p_{1}^{\prime}, \Gamma}\left|\frac{\partial v}{\partial x_{i}}\right|_{p_{1}, \Gamma} \\
=\frac{1}{p_{2}-1} \sum_{i=1}^{n}\left|\left(\int_{\Omega} K(x, y) u_{m}(y) d y\right)^{p(x)-1}\right|_{p_{1}^{\prime}, \Gamma}\left|\int_{\Omega} \frac{\partial}{\partial x_{i}} K(x, y) v(y) d y\right|_{p_{1}, \Gamma} \\
\leq\left.\left.\left.\left. C \sum_{i=1}^{n}\left|K(x)^{p(x)-1}\right| u_{m}\right|_{p_{1}} ^{p(x)-1}\right|_{p_{1}^{\prime}, \Gamma}\left|K_{i}(x)\right| v\right|_{p_{1}}\right|_{p_{1}, \Gamma} \\
\leq C \sum_{i=1}^{n}\left|K(x)^{p(x)-1}\right|_{p_{1}^{\prime}, \Gamma}\left|K_{i}(x)\right|_{p_{1}, \Gamma}\left|u_{m}\right|_{p_{1}}^{p(x)-1}|v|_{p_{1}} \\
\leq C \max \left(\sum_{i=1}^{n}\left|K(x)^{p_{1}-1}\right|_{p_{1}^{\prime}, \Gamma}, \sum_{i=1}^{n}\left|K(x)^{p_{2}-1}\right|_{p_{1}^{\prime}, \Gamma}\right) \\
\quad \times \max \left(\left|u_{m}\right|_{p_{1}}^{p_{1}-1},\left|u_{m}\right|_{p_{1}}^{p_{2}-1}\right)\left|K_{i}(x)\right|_{p_{1}, \Gamma}|v(y)|_{H^{r}}
\end{gathered}
$$

So we have

$$
\begin{gathered}
\left.\left.\quad\left|\int_{\Gamma} \sum_{i=1}^{n} \frac{1}{p(x)-1}\right| u_{m}\right|^{p(x)-2} u_{m} d \Gamma\right|_{H^{-r}(\Omega)} \\
\leq C \max \left(\sum_{i=1}^{n}\left|K(x)^{p_{1}-1}\right|_{p_{1}^{\prime}, \Gamma}, \sum_{i=1}^{n}\left|K(x)^{p_{2}-1}\right|_{p_{1}^{\prime}, \Gamma}\right) \\
\times \max \left(\left|u_{m}\right|_{p_{1}}^{p_{1}-1},\left|u_{m}\right|_{p_{1}}^{p_{2}-1}\right)\left|K_{i}(x)\right|_{p_{1}, \Gamma}<\infty
\end{gathered}
$$

consequently, $\int_{\Gamma} \sum_{i=1}^{n} \frac{1}{p(x)-1}\left|u_{m}\right|^{p(x)-2} u_{m} d \Gamma$ is bounded in $L^{p_{2}^{\prime}}\left(0, T ; H^{-r}(\Omega)\right)$. 
Next, consider $\int_{\Omega} \frac{1}{p(x)-1}\left|u_{m}\right|^{p(x)-2} u_{m} \Delta v d x$, by the same manner, we have

$$
\begin{gathered}
\left.\left.\left|\int_{\Omega} \frac{1}{p(x)-1}\right| u_{m}\right|^{p(x)-2} u_{m} \Delta v d x\left|\leq \frac{1}{p_{1}-1}\right|\left|u_{m}\right|^{p(x)-1}\right|_{p_{2}^{\prime}}|\Delta v|_{p_{2}} \\
\leq\left.\left. C|| u_{m}\right|^{p(x)-1}\right|_{p^{\prime}(\cdot)}|\Delta v|_{p_{2}} \\
\leq C \max \left(\left(\int_{\Omega}\left|u_{m}\right|^{p(x)} d x\right)^{\frac{1}{p_{1}^{\prime}}},\left(\int_{\Omega}\left|u_{m}\right|^{p(x)} d x\right)^{\frac{1}{p_{2}^{\prime}}}\right)|v|_{H^{r}}
\end{gathered}
$$

therefore,

$$
\int_{\Omega} \frac{1}{p(x)-1}\left|u_{m}\right|^{p(x)-2} u_{m} \Delta v d x
$$

is bounded in $L^{p_{2}^{\prime}}\left(0, T ; H^{-r}(\Omega)\right)$. Since $f \in L^{p_{2}^{\prime}}\left(0, T ; L^{p_{2}^{\prime}}(\Omega)\right) \subset L^{p_{2}^{\prime}}\left(0, T ; H^{-r}(\Omega)\right)$, from this discussion and (4.21) it yields that $\frac{\partial}{\partial t} u_{m}$ is bounded in $L^{p_{2}^{\prime}}\left(0, T ; H^{-r}(\Omega)\right)$.

Theorem 4.4. Let $B, B_{1}$ be Banach spaces, and $S$ be a set. Define

$$
M(v)=\max \left(\left(\sum_{i=1}^{n} \int_{\Omega}|v|^{p_{1}-2}\left(\frac{\partial v}{\partial x_{i}}\right)^{2} d x\right)^{\frac{1}{p_{1}}},\left(\sum_{i=1}^{n} \int_{\Omega}|v|^{p_{2}-2}\left(\frac{\partial v}{\partial x_{i}}\right)^{2} d x\right)^{\frac{1}{p_{2}}}\right)
$$

on $S$ with:

$$
\begin{gathered}
\text { a) } S \subset B \subset B_{1} \text {, and } M(v) \geq 0 \text { on } S, \\
M(\lambda v)=\max \left(\left(\sum_{i=1}^{n} \int_{\Omega}|v|^{p_{1}-2}\left(\frac{\partial v}{\partial x_{i}}\right)^{2} d x\right)^{\frac{1}{p_{1}}},\left(\sum_{i=1}^{n} \int_{\Omega}|v|^{p_{2}-2}\left(\frac{\partial v}{\partial x_{i}}\right)^{2} d x\right)^{\frac{1}{p_{2}}}\right) \\
=|\lambda| M(v)
\end{gathered}
$$

b) the set $\{v \mid v \in S, M(v) \leq 1\}$ is relatively compact in $B$.

Define the set

$$
F=\left\{\begin{array}{c}
v: v \text { is locally summable on }[0, T] \text { with value in } B_{1} ; \\
\int_{0}^{T}(M(v(t)))^{q_{0}} d t \leq C, v^{\prime} \text { bounded in } L^{q_{1}}\left(0, T ; B_{1}\right),
\end{array}\right\}
$$

where $1<q_{i}<\infty, i=0,1$. Then $F \subset L^{q_{0}}(0, T ; B)$, and $F$ is relatively compact in $L^{q_{0}}(0, T ; B)$.

We need Theorem (4.4) to prove the following lemma (4.5).

Lemma 4.5. Let $u_{m}$, constructed as in (4.5), be the approximate solution of (1.1)(1.3), then $u_{m} \rightarrow u$ in $L^{p_{2}}\left(0, T ; L^{p_{2}}(\Omega)\right)$ strongly and almost everywhere.

Proof. Let

$$
S=\left\{v: \max \left(|v|^{\frac{p_{1}-2}{2}} v,|v|^{\frac{p_{2}-2}{2}} v\right) \in H^{1}(\Omega)\right\}
$$

Since $H^{1}(\Omega)$ is compactly embedded in $L^{2}(\Omega)$, the proof of [16, Proposition 12.1,p. 143] also works for both $|v|^{\frac{p_{1}-2}{2}} v$ and $|v|^{\frac{p_{2}-2}{2}} v$, then (b) holds. 
Let $B=L^{p_{2}}(\Omega), B_{1}=H^{-r}(\Omega), q_{0}=p_{2}, q_{1}=p_{2}^{\prime}$, we have

$$
\begin{gathered}
\int_{0}^{T}(M(v(t)))^{q_{0}} d t \leq C \int_{0}^{T} \max \left(\begin{array}{c}
\left(\sum_{i=1}^{n} \int_{\Omega}|v|^{p_{1}-2}\left(\frac{\partial v}{\partial x_{i}}\right)^{2} d x\right)^{\frac{p_{2}}{p_{1}}}, \\
\left(\sum_{i=1}^{n} \int_{\Omega}|v|^{p_{2}-2}\left(\frac{\partial v}{\partial x_{i}}\right)^{2} d x\right)
\end{array}\right) d t \\
\leq C \int_{0}^{T} \max \left(\begin{array}{c}
\left(\sum_{i=1}^{n} \int_{\Omega}\left(\frac{\partial}{\partial x_{i}}\left(|v|^{\frac{p_{1}-2}{2}} v\right)\right)^{2} d x\right)^{\frac{p_{2}}{p_{1}}}, \\
\left(\sum_{i=1}^{n} \int_{\Omega}\left(\frac{\partial}{\partial x_{i}}\left(|v|^{\frac{p_{2}-2}{2}} v\right)\right)^{2} d x\right)
\end{array}\right) d t<\infty
\end{gathered}
$$

Now with Lemma (4.3) and a priori estimates, conclusion follows easily from application of Theorem (4.4).

Next, we prove that we can pass the limit in (4.21). Lemmas (4.6)-(4.10), below, show that we can pass the limit in each term in the left-hand side of (4.21)

Lemma 4.6. Let $u_{m}$, constructed as in (4.5), be the approximate solution of (1.1)(1.3), then $\left(\left|u_{m}\right|^{p(x)-2} u_{m}, v\right) \rightarrow\left(|u|^{p(x)-2} u, v\right)$ as $m \rightarrow \infty$.

Proof. Since $u_{m}$ is bounded in $L^{p(\cdot)}(\Omega \times(0, T))$ then $\left|u_{m}\right|^{p(\cdot)-2} u_{m}$ is bounded in $L^{\frac{p(\cdot)}{p(\cdot)-1}}(\Omega \times(0, T))$; hence, using same arguments as in [16, Lemma 1.3], we have

$$
\left|u_{m}\right|^{p(\cdot)-2} u_{m} \rightarrow|u|^{p(\cdot)-2} u \text { weakly in } L^{\frac{p(\cdot)}{p(\cdot)-1}}(\Omega \times(0, T)) .
$$

Lemma 4.7. Let $u_{m}$, constructed as in (4.5), be the approximate solution of (1.1)(1.3), then

$$
\int_{\Gamma} \sum_{i=1}^{n} \frac{1}{p(x)-1}\left(\left|u_{m}\right|^{p(x)-2} \frac{\partial}{\partial x_{i}} u_{m}\right) v d \Gamma \rightarrow \int_{\Gamma} \sum_{i=1}^{n} \frac{1}{p(x)-1}\left(|u|^{p(x)-2} \frac{\partial}{\partial x_{i}} u\right) v d \Gamma
$$

as $m \rightarrow \infty$.

Proof. By a priori estimates, $u_{m}$ is bounded in $L^{p(\cdot)}(\Omega)$ for almost every $t$, then there exists subsequence of $u_{m}$, still denoted as $u_{m}$, converges to $u_{m}$ weak star in $L^{p(\cdot)}(\Omega)$ (Alaoglu's Theorem) for almost every $t \in[0, T]$. Under the assumption that for fixed $x \in \Gamma$, we have

$$
\int_{\Omega} K(x, y) u_{m}(y) d y \rightarrow \int_{\Omega} K(x, y) u(y) d y \text { as } m \rightarrow \infty
$$

Similarly

$$
\int_{\Omega} \frac{\partial}{\partial x_{i}} K(x, y) u_{m}(y) d y \rightarrow \int_{\Omega} \frac{\partial}{\partial x_{i}} K(x, y) u(y) d y \text { as } m \rightarrow \infty
$$

Therefore, for $x \in \Gamma$, we have

$$
\left|u_{m}\right|^{p(\cdot)-2} \frac{\partial}{\partial x_{i}} u_{m} \rightarrow|u|^{p(\cdot)-2} \frac{\partial}{\partial x_{i}} u \text { a.e. }
$$


Since

$$
\begin{gathered}
\max \left(\int_{\Gamma} K^{p_{1}}(x) d \Gamma, \int_{\Gamma} K^{p_{2}}(x) d \Gamma\right)<\infty, \\
\text { and } \max \left(\int_{\Gamma} K_{i}^{p_{1}}(x) d \Gamma, \int_{\Gamma} K_{i}^{p_{2}}(x) d \Gamma\right)<\infty,
\end{gathered}
$$

we have

$$
\left|u_{m}\right|_{p(\cdot), \Gamma} \leq C \max \left(\int_{\Gamma} K^{p_{1}}(x) d \Gamma, \int_{\Gamma} K^{p_{2}}(x) d \Gamma\right) \max \left(\left\|u_{m}\right\|_{p(\cdot)}^{p_{1}},\left\|u_{m}\right\|_{p(\cdot)}^{p_{2}}\right)<\infty
$$

and

$$
\left|\frac{\partial}{\partial x_{i}} u_{m}\right|_{p(\cdot), \Gamma} \leq C \max \left(\int_{\Gamma} K_{i}^{p_{1}}(x) d \Gamma, \int_{\Gamma} K_{i}^{p_{2}}(x) d \Gamma\right) \max \left(\left\|u_{m}\right\|_{p(\cdot)}^{p_{1}},\left\|u_{m}\right\|_{p(\cdot)}^{p_{2}}\right)<\infty .
$$

Then

$$
\begin{gathered}
\left.\left.|| u_{m}\right|^{p(\cdot)-2} \frac{\partial}{\partial x_{i}} u_{m}\right|_{p_{2}^{\prime}, \Gamma} \leq\left.\left. C|| u_{m}\right|^{p(\cdot)-2} \frac{\partial}{\partial x_{i}} u_{m}\right|_{p^{\prime}(\cdot), \Gamma} \operatorname{since}\left(p_{2}^{\prime} \leq p^{\prime}(\cdot) \leq p_{1}^{\prime}\right) \\
\leq\left.\left.|| u_{m}\right|^{p(\cdot)-2}\right|_{\frac{p(\cdot)}{p(\cdot)-2}, \Gamma}\left|\frac{\partial}{\partial x_{i}} u_{m}\right|_{p(\cdot), \Gamma} \operatorname{since}\left(\frac{1}{p^{\prime}(\cdot)}=\frac{p(\cdot)-2}{p(\cdot)}+\frac{1}{p(\cdot)}\right) \\
\leq \max \left(\left(\int_{\Gamma}\left|u_{m}\right|^{p(x)} d \Gamma\right)^{\frac{1}{p_{1}}},\left(\int_{\Omega}\left|u_{m}\right|^{p(x)} d \Gamma\right)^{\frac{1}{p_{2}}}\right) \\
\times \max \left(\left(\int_{\Omega}\left|\frac{\partial}{\partial x_{i}} u_{m}\right|^{p(x)} d \Gamma\right)^{\frac{1}{p_{1}}},\left(\int_{\Gamma}\left|\frac{\partial}{\partial x_{i}} u_{m}\right|^{p(x)} d \Gamma\right)^{\frac{1}{p_{2}}}\right)<\infty .
\end{gathered}
$$

So, applying the same arguments as in [16, Lemma 1.3] to conclude that

$$
\left|u_{m}\right|^{p(\cdot)-2} \frac{\partial}{\partial x_{i}} u_{m} \rightarrow|u|^{p(\cdot)-2} \frac{\partial}{\partial x_{i}} u \text { weakly in } L^{p_{2}^{\prime}}(\Gamma) .
$$

for a.e. $t \in[0, T]$. Since,

$$
\max \left(\left(\int_{\Omega}\left|\frac{\partial}{\partial x_{i}} v\right|^{p(x)} d \Gamma\right)^{\frac{1}{p_{1}}},\left(\int_{\Omega}\left|\frac{\partial}{\partial x_{i}} v\right|^{p(x)} d \Gamma\right)^{\frac{1}{p_{2}}}\right)<\infty,
$$

the proof is complete.

Lemma 4.8. Let $u_{m}$, constructed as in (4.5), be the approximate solution of (1.1)(1.3), then

$$
\int_{\Gamma} \sum_{i=1}^{n} \frac{1}{p(x)-1}\left|u_{m}\right|^{p(x)-2} u_{m} \frac{\partial v}{\partial x_{i}} d \Gamma \rightarrow \int_{\Gamma} \sum_{i=1}^{n} \frac{1}{p(x)-1}|u|^{p(x)-2} u \frac{\partial v}{\partial x_{i}} d \Gamma
$$

as $m \rightarrow \infty$. 
Proof. From the proof of Lemma (4.7), we have, for $x \in \Gamma,\left|u_{m}\right|^{p(\cdot)-2} u_{m} \rightarrow|u|^{p(\cdot)-2} u$ almost everywhere, and

$$
\begin{gathered}
\left.\left.|| u_{m}\right|^{p(\cdot)-2} u_{m}\right|_{p_{2}^{\prime}, \Gamma} \leq\left.\left. C|| u_{m}\right|^{p(\cdot)-2} u_{m}\right|_{p^{\prime}(\cdot), \Gamma} \\
\leq \max \left(\left(\int_{\Gamma}\left|u_{m}\right|^{p(x)} d \Gamma\right)^{\frac{1}{p_{1}}},\left(\int_{\Gamma}\left|u_{m}\right|^{p(x)} d \Gamma\right)^{\frac{1}{p_{2}}}\right)<\infty .
\end{gathered}
$$

Therefore, by applying [16, Lemma 1.3] we conclude that

$$
\left|u_{m}\right|^{p(\cdot)-2} u_{m} \rightarrow|u|^{p(\cdot)-2} u \text { weakly in } L^{p_{2}^{\prime}}(\Gamma) .
$$

Since $\frac{\partial v}{\partial x_{i}} \in L^{p_{2}^{\prime}}(\Gamma)$, the proof is complete.

Lemma 4.9. Let $u_{m}$, constructed as in (4.5), be the approximate solution of (1.1)(1.3), then

$$
\int_{\Omega} \frac{1}{p(x)-1}\left(\left|u_{m}\right|^{p(x)-2} u_{m}\right) \Delta v d x \rightarrow \int_{\Omega} \frac{1}{p(x)-1}\left(|u|^{p(x)-2} u\right) \Delta v d x
$$

as $m \rightarrow \infty$.

Proof. From lemma $((4.5))$, we have $\left|u_{m}\right|^{p(\cdot)-2} u_{m} \rightarrow|u|^{p(\cdot)-2} u$ almost everywhere, for $x \in \Omega$, since

$$
\begin{gathered}
\left.\left.|| u_{m}\right|^{p(\cdot)-2} u_{m}\right|_{p_{2}^{\prime}, \Omega} \leq\left.\left. C|| u_{m}\right|^{p(\cdot)-2} u_{m}\right|_{p^{\prime}(\cdot), \Omega} \\
\leq \max \left(\left(\int_{\Omega}\left|u_{m}\right|^{p(x)} d x\right)^{\frac{1}{p_{1}}},\left(\int_{\Omega}\left|u_{m}\right|^{p(x)} d x\right)^{\frac{1}{p_{2}}}\right)<\infty .
\end{gathered}
$$

by $[16$, Lemma 1.3$]$, we have $\left|u_{m}\right|^{p(\cdot)-2} u_{m} \rightarrow|u|^{p(\cdot)-2} u$ weakly in $L^{p_{2}^{\prime}}(\Omega)$. Since $\Delta v \in L^{p_{2}}(\Omega)$, the proof is complete.

Lemma 4.10. Let $u_{m}$, constructed as in (4.5), be the approximate solution of (1.1)(1.3), then

as $m \rightarrow \infty$.

$$
\left(\sum_{i=1}^{n}\left(\left|u_{m}\right|^{p(x)-2} \frac{\partial u_{m}}{\partial x_{i}}\right), \frac{\partial}{\partial x_{i}} v\right) \rightarrow\left(\sum_{i=1}^{n}\left(|u|^{p(x)-2} \frac{\partial u}{\partial x_{i}}\right), \frac{\partial}{\partial x_{i}} v\right)
$$

Proof. Replacing the results of (4.8) and (4.9) in (4.22), the proof is complete.

Lemma 4.11. Let $u_{m}$, constructed as in (4.5). be the approximate solution of (1.1)(1.3), then $\left(\frac{\partial}{\partial t} u_{m}, v\right) \rightarrow\left(\frac{\partial}{\partial t} u, v\right)$ and $u(t)$ is continuous on $[0, T]$.

Proof. Since $\frac{\partial}{\partial t} u_{m}(t)$ is bounded in $L^{p_{2}^{\prime}}\left(0, T ; H^{-r}(\Omega)\right)$, by Alaoglu's theorem, there exists a subsequence, still denoted by $\frac{\partial}{\partial t} u_{m}(t)$, converging to $\chi$ weak star in $L^{p_{2}^{\prime}}\left(0, T ; H^{-r}(\Omega)\right)$. By slightly modifying the proof of $[6$, Theorem 1$]$ (with the space $L^{p_{2}^{\prime}}\left(0, T ; H^{-r}(\Omega)\right)$ instead of $L^{2}\left(0, T ; B_{2}^{1}(0,1)\right)$.), we have $\chi=u^{\prime}$ and $u$ is continuous on $[0, T]$. This ends the proof of Lemma (4.11).

Combining all above results, the existence theorem (4.1) follows. 
Acknowledgements. The authors would like to thank the anonymous referees and the handling editor for their careful reading and for relevant remarks/suggestions which helped him to improve the paper.

\section{References}

[1] Aboulaich, R., Meskine, D., Souissi, A., New diffusion models in image processing, Comput. Math. Appl., 56(2008), no. 4, 874-882.

[2] Adams, R.A., Sobolev Spaces, Academic Press, 2003.

[3] Antontsev, S.N., Shmarev, S.I., Elliptic Equations with Anisotropic Nonlinearity and Nonstandard Growth Conditions, Handbook of Differential Equations, Stationary Partial Differential Equations, vol. 3, 2006.

[4] Antontsev, S.N., Shmarev, S.I., Blow-up of solutions to parabolic equations with nonstandard growth conditions, J. Comput. Appl. Math., 234(2010), no. 9, 2633-2645.

[5] Antontsev, S.N., Zhikov, V., Higher integrability for parabolic equations of $p(x, t)$ Laplacian type, Adv. Differential Equations, 10(2009), no. 9, 1053-1080.

[6] Bouziani, A., Merazga, N., Benamira, S., Galerkin method applied to a parabolic evolution problem with nonlocal boundary conditions, Nonlinear Analysis, 69(2008), 15151524 .

[7] Chen, B., Existence of solutions for quasilinear parabolic equations with nonlocal boundary conditions, Electronic Journal of Differential Equations, 2011(2011), no. 18, 1-9.

[8] Chen, Y., Levine, S., Rao, M., Variable exponent, linear growth functionals in image restoration, SIAM J. Appl. Math., 66(2006), 1383-1406.

[9] Diening, L., Hästo, P., Harjulehto, P., Ruz̃icka, M., Lebesgue and Sobolev Spaces with Variable Exponents, in: Springer Lecture Notes, Springer-Verlag, vol. 2017, Berlin, 2011.

[10] Diening, L., Ruz̃icka, M., Calderon Zygmund operators on generalized Lebesgue spaces $L^{p(x)}(\Omega)$ and problems related to fluid dynamics, J. Reine Angew. Math., 563(2003), $197-220$.

[11] Diening, L., Ruz̃icka, M., Calderon Zygmund operators on generalized Lebesgue spaces $L^{p(x)}(\Omega)$ and problems related to fluid dynamics, Preprint Mathematische Fakultät, Albert-Ludwigs-Universität Freiburg, 120(2002), 197-220.

[12] Fan, X., Shen, J., Zhao, D., Sobolev embedding theorems for spaces $W^{k, p(x)}(\Omega)$, J. Math. Anal. Appl., 262(2001), 749-760.

[13] Fu, Y., The existence of solutions for elliptic systems with nonuniform growth, Studia Math., 151(2002), 227-246.

[14] Kovàcik, O., Rákosnik, J., On spaces $L^{p(x)}$ and $W^{1, p(x)}(\Omega), 41(1991)$.

[15] Lian, S., Gao, W., Cao, C., Yuan, H., Study of the solutions to a model porous medium equation with variable exponent of nonlinearity, J. Math. Anal. Appl., 342(2008), no. 1, 27-38.

[16] Lions, J.L., Quelques méthodes de résolution des problèmes aux limites non linéaires, Dunod, Paris, 1966.

[17] Rahmoune, A., Semilinear Hyperbolic Boundary Value Problem Associated to the Nonlinear Generalized Viscoelastic Equations, Acta Mathematica Vietnamica, 43(2018), no. 2, 219-238. 
[18] Rahmoune, A., On the existence, uniqueness and stability of solutions for semilinear generalized elasticity equation with general damping, Acta Mathematica Sinica, English Series, 33(2017), no. 11, 1549-1564.
Abita Rahmoune
Department of Technical Sciences
03000 Laghouat University, Algeria
e-mail: abitarahmoune@yahoo.fr
Benyattou Benabderrahmane
Laboratory of Pure and Applied Mathematics
Mohamed Boudiaf Université - M'Sila, Algeria
e-mail: bbenyattou@yahoo.com 